This item was submitted to Loughborough's Research Repository by the author.

Items in Figshare are protected by copyright, with all rights reserved, unless otherwise indicated.

\title{
Inertial oscillations and frontogenesis driven by a quadratic horizontal density
} variation

PLEASE CITE THE PUBLISHED VERSION

LICENCE

CC BY-NC-ND 4.0

\section{REPOSITORY RECORD}

Kay, Anthony. 2019. "Inertial Oscillations and Frontogenesis Driven by a Quadratic Horizontal Density Variation”. figshare. https://hdl.handle.net/2134/245. 


\title{
Inertial oscillations and frontogenesis driven by a quadratic horizontal density variation
}

\author{
A. KAY \\ Department of Mathematical Sciences, Loughborough University, \\ Loughborough, Leicestershire, LE11 3TU
}

Tel: +44 1509222878

Fax: +44 1509223969

Email: A.Kay@Lboro.ac.uk 


\begin{abstract}
We consider inviscid rotating flow driven by a horizontally quadratic density variation in a horizontally unbounded slab. This configuration permits a similarity solution, removing the dependence on the horizontal coordinate from the vorticity and temperature equations, which are then solved by numerical integration along characteristics. At large values of Rossby number, the flow proceeds to a singularity in a similar manner to the non-rotating flow with the same initial conditions. At small values of Rossby number there are inertial oscillations of growing amplitude, which have been analysed using the method of multiple scales. The oscillations become desynchronised between the upper and lower parts of the domain, and static instability appears for a small fraction of each oscillation period. Eventually the oscillations give way to the rapid formation of a singularity, in contrast to geostrophic adjustment theory which predicts that a singularity will form only if the Rossby number is sufficiently large.
\end{abstract}

Keywords: inertial oscillations, frontogenesis, multiple scales, geostrophic adjustment

\title{
1 Introduction
}

The role of curvature in the horizontal density profile $\left(\partial^{2} \rho / \partial x^{2} \neq 0\right)$ as a cause of frontogenesis has been established by $\mathrm{Ou}$ (1984) for rotating flow on an $f$-plane and by Simpson and Linden (1989) in the absence of coriolis forces. For rotating flow, geostrophic adjustment theory shows that the final equilibrium state depends on the maximum value of $\partial^{2} \rho / \partial x^{2}$ present in the initial configuration (Ou, 1984; Blumen and $\mathrm{Wu}, 1995)$ : for small values of this parameter, a flow in geostrophic equilibrium with some steepening of density gradients is obtained, but above a critical initial value of $\partial^{2} \rho / \partial x^{2}$, a singularity appears and this is interpreted as a front. Geostrophic 
adjustment theory finds an equilibrium state with the same energy as the initial unbalanced state, but in the absence of damping the system will display inertial oscillations about its equilibrium, never settling down to a steady state (Rhines, 1988; Tandon and Garrett, 1994). This motivated Blumen (2000) (hereafter denoted BL) to solve a time-dependent extension of the Blumen and Wu (1995) problem, obtaining either inertial oscillations about geostrophic equilibrium or else the formation of a singularity (front) within the first half of an oscillation period, again dependent on the initial value of $\partial^{2} \rho / \partial x^{2}$. Subsequently Blumen and Williams (2001) (hereafter BW) generalised the earlier work to include the effects of a barotropic pressure gradient, and found in their numerical simulations that at the end of one inertial oscillation period the system had not returned precisely to its initial state.

Studies of frontogenesis in a non-rotating frame of reference have followed a different, more mathematical, path. In particular, attention has focussed on the case of a quadratic horizontal density variation. Kay (1992) has given a geophysical motivation for this, arising from the approximately quadratic temperature-density relation around the temperature of maximum density in fresh water. Nevertheless, the mathematical simplification arising from this density profile, namely that in a horizontally unbounded domain it admits a similarity solution which reduces the number of space dimensions in the governing equations (Amin and Riley, 1990), is probably a more significant reason for its adoption. Jacqmin (1991) proved that a singularity will form on one of the horizontal boundaries in finite time, starting from initial conditions of a fluid at rest with a quadratic density profile. Grundy and Kay (2003) then gave a detailed analysis of the flow structure at times shortly before the singularity appears. Kay (1992) identified the dynamical process responsible for singularity formation as being a positive feedback: the curved density profile generates a horizontally con- 
vergent flow, and this convergence steepens the density gradient, also increasing the curvature of the density profile; increased curvature then leads to more intense convergence, faster steepening of gradients, and so on.

Apart from the recent work of BW, there has been little recognition of any connection between the strands of research described in the two paragraphs above. The present work will rectify this by applying the mathematical formalism hitherto associated with studies of non-rotating flow to the problem of frontogenesis on an $f$-plane. The quadratic horizontal density variation, which still admits a similarity solution when coriolis terms are included in the equations, will be adopted. The observation by BW that the resultant behaviour "is unrealistic for geophysical applications" is related to the horizontally unbounded nature of the density profile, which leads to infinite values of velocity and density gradient along the entire length of a horizontal boundary, rather than a localised front. Nevertheless, the model can provide useful dynamical insights, and we shall present results for times long before the appearance of the physically unrealistic singularity, displaying features not apparent in any of the studies by Blumen and his collaborators.

Our system of equations will be presented in Section 2, followed by a brief presentation of results from geostrophic adjustment theory for this system in Section 3. The solution of the full time-dependent problem, by numerical methods and by asymptotic analysis, is given in Section 4. The most important results are found in the case of small Rossby number, in which frontogenesis proceeds through the slow growth in amplitude of inertial oscillations. This is unrelated to the slow frontogenesis process described by Blumen and Lundquist (2001), which results from Ekman layer dynamics; there are no viscous or other dissipative effects in the present model. 


\section{The mathematical model}

\subsection{Governing equations}

The coordinate system $(x, y, z)$ with corresponding velocity components $(u, v, w)$ is defined as follows. The $z$ coordinate is vertical, with the fluid being confined between rigid horizontal boundaries at $z=0$ and $z=h$, i.e. with

$$
w=0 \quad \text { at } \quad z=0 \quad \text { and at } \quad z=h .
$$

Imposing an initial density variation in only one horizontal direction, designated $x$, the flow will remain independent of the second horizontal coordinate $y$ at all times. Nevertheless, a velocity normal to the $x-z$ plane is induced by the coriolis force:

$$
\frac{\partial v}{\partial t}+u \frac{\partial v}{\partial x}+w \frac{\partial v}{\partial z}=-f_{z} u+f_{x} w .
$$

Here, $f_{x}$ and $f_{z}$ are components of twice the earth's angular velocity vector; traditionally, only the vertical component $f_{z}$ is retained in oceanic and atmospheric applications, but Sander et al. (1995) have pointed out that horizontal components of planetary rotation should be retained when there are substantial vertical velocities, as can occur in frontal situations.

Under the usual incompressibility assumption, the flow in the $x-z$ plane satisfies the continuity equation

$$
\frac{\partial u}{\partial x}+\frac{\partial w}{\partial z}=0
$$

and has vorticity

$$
\omega \equiv \frac{\partial u}{\partial z}-\frac{\partial w}{\partial x} .
$$

This vorticity evolves according to

$$
\frac{\partial \omega}{\partial t}+u \frac{\partial \omega}{\partial x}+w \frac{\partial \omega}{\partial z}-f_{x} \frac{\partial v}{\partial x}-f_{z} \frac{\partial v}{\partial z}=\frac{g}{\rho_{m}} \frac{\partial \rho}{\partial x},
$$


where the Boussinesq approximation has been used in formulating the baroclinicity term on the right-hand side of (2.5), and $\rho_{m}$ is a constant reference density.

We shall use the approximate equation of state given by Farmer and Carmack (1981) for water of zero salinity near the temperature of maximum density:

$$
\rho=\rho_{m}\left[1+p\left(C_{0}-\gamma\left(\theta-\theta_{m}\right)\right)-\beta\left(\theta-\theta_{m}\right)^{2}\right]
$$

where $p$ is pressure above atmospheric, $\theta$ is temperature and $\theta_{m}=3.98^{\circ} \mathrm{C}$, the temperature of maximum density at $p=0$. The remaining constants in (2.6) have the values

$$
\begin{aligned}
\rho_{m} & =999.975 \mathrm{~kg} \cdot \mathrm{m}^{-3} \\
C_{0} & =4.9388 \times 10^{-10} \mathrm{~Pa}^{-1} \\
\gamma & =3.3039 \times 10^{-12}{ }^{\circ} \mathrm{C}^{-1} \cdot \mathrm{Pa}^{-1} \\
\beta & =8.2545 \times 10^{-6}{ }^{\circ} \mathrm{C}^{-2} .
\end{aligned}
$$

The important point to note for the present study is that both cabbeling $\left(\partial^{2} \rho / \partial \theta^{2}<\right.$ $0)$ and thermobaricity $\left(\partial^{2} \rho / \partial \theta \partial p<0\right)$ are accounted for, in the simplest form possible (both second derivatives of density being constant). In the thermobaric term in (2.6), pressure may be calculated hydrostatically using the reference density:

$$
p=\rho_{m} g(h-z)
$$

Thus the density gradient in (2.5) may be written in terms of temperature and depth as

$$
\frac{\partial \rho}{\partial x}=-\rho_{m}\left\{g \rho_{m} \gamma(h-z)+2 \beta\left(\theta-\theta_{m}\right)\right\} \frac{\partial \theta}{\partial x}
$$


Ignoring compressibility effects, the temperature is conserved:

$$
\frac{\partial \theta}{\partial t}+u \frac{\partial \theta}{\partial x}+w \frac{\partial \theta}{\partial z}=0
$$

Initially the fluid is assumed to be at rest, with a linear temperature variation extending horizontally to $\pm \infty$ :

$$
u=v=w=0, \quad \theta=\theta_{m}+\kappa x \quad \text { at } \quad t=0,
$$

where the origin of the $x$-coordinate is set at the location of maximum density at the upper surface. The contradiction between the unbounded density configuation and the Boussinesq approximation (based on density variations being small), and indeed with the range of validity of (2.6), will be ignored: our focus is on constructing a mathematically elegant model to examine effects of nonlinear horizontal density profiles.

The above model has more in common with that of BL and BW than might be apparent at first sight. If thermobaricity and the horizontal component of planetary rotation are ignored (as they will be in some of our calculations below), the similarities become clearer when we note the following points. Firstly, in the absence of diffusive effects, it makes no difference whether the nonlinear density profile arises through nonlinearity in the equation of state (as here) or in the temperature profile (as in BL and BW). Secondly, the form (2.5) of the vorticity equation (with $f_{x}=0$ ) may be derived from BL and BW's $x$-momentum equation and hydrostatic equation if the latter is formulated in terms of density rather than temperature, provided that $\partial w / \partial x \equiv 0$; but this condition is satisfied by the similarity solution (see below). Thirdly, our model has zero potential vorticity: defining the Ertel's potential vorticity by

$$
q \equiv\left(f_{z}+\frac{\partial v}{\partial x}\right) \frac{\partial \theta}{\partial z}-\frac{\partial v}{\partial z} \frac{\partial \theta}{\partial x}
$$


the conservation law

$$
\frac{\partial q}{\partial t}+u \frac{\partial q}{\partial x}+w \frac{\partial q}{\partial z}=0
$$

can be derived from (2.2) (with $\left.f_{x}=0\right),(2.3)$ and (2.9); the motionless, thermally unstratified initial conditions (2.10) then yield

$$
q=0
$$

However, if potential vorticity were defined in terms of density rather than temperature (Blumen and $\mathrm{Wu}, 1995)$, the inclusion of thermobaricity would render it non-zero and non-uniform.

An important dimensionless parameter, measuring the relative magnitudes of buoyancy and coriolis forces, is the Rossby number defined by BL as

$$
a_{B L}=\frac{\left(g^{*} h\right)^{1 / 2}}{f_{z} L} .
$$

Here $g^{*}=g \Delta \rho / \rho_{m}$, where $\Delta \rho$ is a characteristic magnitude of density variations and $L$ is their characteristic horizontal length-scale. With our unbounded density configuration neither $\Delta \rho$ nor $L$ can be defined separately, but from (2.6) and (2.10) we have $\Delta \rho / L^{2} \sim \rho_{m} \beta \kappa^{2}$. We therefore define our Rossby number as

$$
a=\frac{\kappa \sqrt{2 \beta g h}}{f_{z}}
$$

(where the factor of 2 is for later convenience). A useful interpretation of this parameter is as the ratio of the time-scales for rotation and for frontogenesis (where the latter is given by Kay (1992)).

\subsection{The similarity solution}

Similarity solutions for stagnation-point flows in domains which are unbounded in one dimension (say $x$ ) are well-known (e.g. Proudman and Johnson (1962)): each term 
in the Navier-Stokes or vorticity equation is proportional to $x$, so the $x$-dependence can be extracted. Amin and Riley (1990) pointed out that a quadratic density profile introduces a density gradient term also proportional to $x$, so retaining the similarity solution. In a rotating frame, the terms involving $f_{z}$ again preserve the similarity property in both (2.5) and (2.2). However, the terms involving $f_{x}$ and also those arising from thermobaricity in the density gradient are independent of $x$. Kay (2001) showed how to construct a similarity solution when such terms are present: we now extend the development in that paper to include coriolis terms.

Defining the dimensionless parameter

$$
b=\frac{\gamma g \rho_{m}}{2 \beta \kappa},
$$

which indicates the relative importance of thermobaricity and nonlinear temperaturedependence in the density field, we make the ansatz:

$$
\begin{aligned}
\theta & =\theta_{m}+\kappa\left(x \Theta(Z, T)+b h \Theta_{t}(Z, T)\right) \\
\omega & =\frac{(2 \beta g)^{1 / 2} \kappa}{h^{1 / 2}}\left(x \Omega(Z, T)+b h \Omega_{t}(Z, T)\right) \\
u & =(2 \beta g h)^{1 / 2} \kappa\left(x U(Z, T)+b h U_{t}(Z, T)\right) \\
v & =(2 \beta g h)^{1 / 2} \kappa\left(x V(Z, T)+b h V_{t}(Z, T)\right) \\
w & =\left(2 \beta g h^{3}\right)^{1 / 2} \kappa W(Z, T)
\end{aligned}
$$

in which upper-case letters indicate dimensionless variables, with

$$
Z=\frac{z}{h}
$$

and

$$
T=(2 \beta g h)^{1 / 2} \kappa t
$$


and subscripts $t$ indicate contributions to dependent variables from thermobaric effects. Note that according to (2.21), $\partial w / \partial x \equiv 0$.

We now substitute for the density gradient from (2.8) into the vorticity equation (2.5), then substitute from (2.17) - (2.21) into this and also into the normal velocity equation (2.2) and the temperature equation (2.9). Separating terms proportional to $x$ from those independent of $x$ and introducing a further dimensionless parameter

$$
c=\frac{f_{x}}{f_{z}},
$$

we obtain a set of six partial differential equations in $Z$ and $T$ :

$$
\begin{aligned}
\frac{\partial \Omega}{\partial T} & =-U \Omega-W \frac{\partial \Omega}{\partial Z}+\frac{1}{a} \frac{\partial V}{\partial Z}-\Theta^{2} \\
\frac{\partial \Omega_{t}}{\partial T} & =-U_{t} \Omega-W \frac{\partial \Omega_{t}}{\partial Z}+\frac{1}{a}\left(\frac{c}{b} V+\frac{\partial V_{t}}{\partial Z}\right)+\left(Z-1-\Theta_{t}\right) \Theta \\
\frac{\partial V}{\partial T} & =-U V-W \frac{\partial V}{\partial Z}-\frac{1}{a} U \\
\frac{\partial V_{t}}{\partial T} & =-U_{t} V-W \frac{\partial V_{t}}{\partial Z}+\frac{1}{a}\left(\frac{c}{b} W-U_{t}\right) \\
\frac{\partial \Theta}{\partial T} & =-U \Theta-W \frac{\partial \Theta}{\partial Z} \\
\frac{\partial \Theta_{t}}{\partial T} & =-U_{t} \Theta-W \frac{\partial \Theta_{t}}{\partial Z} .
\end{aligned}
$$

The continuity equation (2.3) and the definition of vorticity (2.4) yield

$$
\begin{aligned}
\Omega & =\frac{\partial U}{\partial Z}=-\frac{\partial^{2} W}{\partial Z^{2}} \\
\Omega_{t} & =\frac{\partial U_{t}}{\partial Z} .
\end{aligned}
$$

The initial conditions are

$$
W=U=U_{t}=\Omega=\Omega_{t}=V=V_{t}=0, \quad \Theta=1, \quad \Theta_{t}=0 \quad \text { at } \quad t=0
$$


and the boundary conditions are

$$
W=0 \quad \text { at } \quad Z=0 \text { and at } Z=1 .
$$

The following observations may be made concerning the system of equations (2.25) (2.34):-

(i) The first-order equations (2.25) - (2.30) may be solved in terms of characteristics:

$$
\begin{aligned}
\mathrm{d} T & =\frac{\mathrm{d} Z}{W}=\frac{\mathrm{d} \Omega}{-U \Omega-\Theta^{2}+a^{-1} \partial V / \partial Z} \\
& =\frac{\mathrm{d} \Omega_{t}}{-U_{t} \Omega+Z \Theta-\Theta-\Theta \Theta_{t}+a^{-1}\left((c / b) V+\partial V_{t} / \partial Z\right)}=\frac{\mathrm{d} V}{-U\left(V+a^{-1}\right)} \\
& =\frac{\mathrm{d} V_{t}}{-U_{t} V+a^{-1}\left((c / b) W-U_{t}\right)}=\frac{\mathrm{d} \Theta}{-U \Theta}=\frac{\mathrm{d} \Theta_{t}}{-U_{t} \Theta} .
\end{aligned}
$$

This formulation is useful for numerical solution of the equations.

(ii) The evolution of the unsubscripted variables $\Omega, V$ and $\Theta$ is not affected by any of the $t$-subscripted variables. The effects of thermobaricity and the horizontal component of planetary rotation $f_{x}$ are confined to the latter variables (through the splitting in $(2.17)$ - (2.20) which involves the thermobaricity parameter $b$, and through the appearance of the coriolis ratio $c$ only in equations for the $t$-subscripted variables). Thus the time and vertical location of frontogenesis depend solely on the nonlinearity of the density profile and on $f_{z}$; thermobaricity and $f_{x}$ can only influence the detailed structure of the flow (Kay, 2001). Note that if it was required to include the effects of $f_{x}$ but to exclude thermobaricity, an alternative splitting would need to be employed in (2.17) - (2.20), with the coriolis ratio $c$ replacing the thermobaricity parameter $b$; the structure of the resulting equations would remain similar to that of $(2.25)-(2.30)$. 
(iii) The zero potential vorticity condition can be expressed by the identities

$$
\Theta=1+a V, \quad \Theta_{t}=a V_{t} .
$$

It is easy to verify that substitution of (2.36) into (2.29) and (2.30) yields (2.27) and (2.28) provided that $c=0$ (the definition of potential vorticity excludes consideration of $f_{x}$ ); the initial conditions on $\Theta, \Theta_{t}, V$ and $V_{t}$ are also satisfied by (2.36). The physical significance of the identities is revealed by expressing $q$ from (2.11) in terms of the dimensionless variables and noting that equations (2.36) are sufficient conditions for $q=0$.

(iv) Another conserved quantity is the integral

$$
I_{0}=\int_{0}^{1} \frac{1}{\Theta} \mathrm{d} Z,
$$

(from (2.29), (2.31) and the boundary conditions (2.34): see Grundy and Kay (2003)). With the initial conditions (2.33) its value is $I_{0}=1$. The physical meaning of this quantity is not clear, but it has some of the character of an entropy: in particular, if viscous and diffusive terms are included in the governing equations, $I_{0}$ becomes a monotonic (decreasing) function of time.

The above formulation will be used in our studies of the time-dependent problem in Section 4. However, it is instructive to first examine an equilibrium solution, derived by geostrophic adjustment theory, even though the system never settles down to equilibrium.

\section{Geostrophic adjustment in thermobaric flow}

Geostrophic adjustment theory assumes an isentropic transition from an initial unbalanced state (at rest, with horizontal density variations) to a final state in geostrophic 
equilibrium. We use the Lagrangian formalism developed by $\mathrm{Ou}$ (1984); the calculation is simplified by the uniformity of $\partial^{2} \rho / \partial x^{2}$ but complicated by the inclusion of thermobaricity. The theory neglects effects due to the horizontal component of planetary rotation; note that setting $f_{x}=0$ is equivalent to supposing that the temperature gradient is zonal.

In geostrophic equilibrium the buoyancy force balances the coriolis force due to the vertical component of the earth's rotation:

$$
f_{z} \frac{\partial v_{g}}{\partial z}=g \frac{\partial \rho}{\partial x}
$$

(where we use subscripts $g$ throughout to denote quantities in geostrophic equilibrium). In the Lagrangian analysis, a particle is identified by its initial coordinates $(\xi, \zeta)$; its equilibrium position $\left(x_{g}, z_{g}\right)$ is then given by

$$
\frac{\partial x_{g}}{\partial \zeta}=\frac{g}{\rho_{m} f_{z}^{2}}\left(\frac{\partial \rho}{\partial \xi} \frac{\partial z_{g}}{\partial \zeta}-\frac{\partial \rho}{\partial \zeta} \frac{\partial z_{g}}{\partial \xi}\right) .
$$

Further analysis is intractable unless the second term in parentheses in (3.2) vanishes. This occurs if there is no initial density stratification, and also if the vertical displacement of particles is independent of their initial horizontal position. The similarity solution shows that $\partial w / \partial x=0$ (as a result of the horizontal uniformity of $\partial^{2} \rho / \partial x^{2}$ ); thus $\partial z_{g} / \partial \xi=0$, so that the density stratification brought about by thermobaricity does not affect the solution. This not only simplifies (3.2), but also reduces the Lagrangian form of the continuity equation to

$$
\frac{\partial x_{g}}{\partial \xi} \frac{\partial z_{g}}{\partial \zeta}=1
$$

Proceeding as in $\mathrm{Ou}(1984)$ we obtain the equilibrium particle positions and geostrophic velocity in terms of the Rossby number a defined in (2.15):

$$
z_{g}=\frac{h}{a^{2}}\left(1+\frac{a^{2}}{2}-\sqrt{\left(1+\frac{a^{2}}{2}\right)^{2}-2 a^{2} \frac{\zeta}{h}}\right)
$$


(confirming that $z_{g}$ is independent of $\xi$ );

$$
x_{g}=\left(1+\frac{a^{2}}{2}-a^{2} \frac{z_{g}}{h}\right) \xi+C
$$

and

$$
v_{g}=-f_{z}\left(x_{g}-\xi\right)=f_{z}\left(a^{2}\left(\frac{z_{g}}{h}-\frac{1}{2}\right) \xi-C\right)
$$

where $C$ is a constant, dependent on Rossby number with $C=O\left(a^{2}\right)$ as $a \rightarrow 0$.

The particle displacements and velocity depend on the curvature of the initial horizontal density profile (see $\mathrm{Ou}$ (1984) and note that $a^{2} \propto \beta$, where $\beta$ is the nonlinearity parameter in the equation of state (2.6)), but not on the thermobaricity parameter $\gamma$. However, thermobaricity does come in through the constant horizontal displacement $C$. For non-rotating flow, Kay (2001) found that thermobaricity displaced the axis of symmetry of a front by an amount that could not be determined without solving an initial-value problem; similarly in the present case, symmetry considerations in the absence of thermobaricity yield $C=0$, but thermobaricity breaks the symmetry and leaves $C$ undetermined. Adopting the formalism of Blumen and $\mathrm{Wu}$ (1995) does not appear to resolve this difficulty, since these authors also appeal to symmetry in their zero potential vorticity solution.

Horizontal convergence of fluid particles is indicated by values of $\partial x_{g} / \partial \xi$ less than unity. From (3.5), there is a pattern of convergence in the upper half of the flow domain $\left(z_{g}>h / 2\right)$ and divergence in the lower half. Thus vertical displacements are downward everywhere: from (3.4), we find for $a<<1$ (coriolis forces dominant) that

$$
z_{g}-\zeta \approx-a^{2} \frac{\zeta}{2}\left(1-\frac{\zeta}{h}\right)
$$

while for $a=\sqrt{2}$ the maximum vertical displacement is for particles which arrive at $z_{g}=h / 2$, having descended from $\zeta=3 h / 4$. The case $a=\sqrt{2}$ is of significance 
as being the lowest value of Rossby number for which a front can form. This is determined by noting that if $\partial x_{g} / \partial \xi \leq 0$, particles are unphysically occupying the same space or passing through each other, which is interpreted as indicating front formation (Ou, 1984). From (3.5), a front appears at the upper surface $(z=h)$ if $a \geq \sqrt{2}$, i.e. if $\partial^{2} \rho / \partial \xi^{2} \geq 2 f_{z}^{2} \rho_{m} / g h$. However, because of the uniformity of $\partial^{2} \rho / \partial \xi^{2}$, Ou's analysis of the frontal position breaks down; instead we find a rather unphysical process of uniform blow-up along the upper surface (Kay, 1992).

There is an internal contradiction in geostrophic adjustment theory (which does not prevent it from providing useful insights): the isentropic assumption is based on the idea that the system settles down to equilibrium quickly; yet the absence of dissipation of mechanical energy means that, like a frictionless pendulum, the isentropic flow can never settle down at all. Instead, it must perform inertial oscillations; these are analysed in the next section.

\section{The time-dependent solution}

BL's analytical solution of their time-dependent problem revealed two possible scenarios. For low Rossby numbers the system performs inertial oscillations, with frontogenesis during the first half-period $0<t<\pi / f_{z}$ being exactly reversed by frontolysis during the second half-period, so that the system returns to its initial state at time $t=2 \pi / f_{z}$. If the Rossby number is above some critical value, a front forms within the first half-period (and the solution cannot be continued beyond the appearance of this singularity). BL ignored the existence of a barotropic pressure gradient; BW included this factor in their numerical solution, and found that frontogenesis was delayed and that inertial oscillations were not exactly periodic (the state at $t=2 \pi / f_{z}$ 
was not identical to the initial state). However, they did not pursue the solution much beyond one oscillation period in the low Rossby number case. The solutions we present below include a feature not found by BL or BW: inertial oscillations of gradually increasing amplitude, eventually leading to the formation of a front (even for low Rossby numbers).

\subsection{Intuitive ideas}

The similarity property of our model means that there is a direct proportionality between density gradient $\partial \rho / \partial x$, which is responsible for generating vorticity, and density profile curvature $\partial^{2} \rho / \partial x^{2}$, which is responsible for horizontal convergence and divergence in the rotational flow. Where the flow is convergent, the density gradient and profile curvature will increase, accelerating the convergence and so leading to rapid frontogenesis: a positive feedback. Where the flow is divergent, the density gradient and profile curvature will decrease, reducing the rate of divergence and so slowing down the frontolysis: a negative feedback. In a non-rotating frame, the result of these processes operating in different parts of the flow domain is a layer of highvorticity flow with very steep density gradients near one horizontal boundary, while the remainder of the flow tends towards irrotationality (Grundy and Kay, 2003). We now consider how planetary rotation modifies the process.

Useful insights can be gained by means of a simple calculation using the method of expanding in powers of $t$ (Simpson and Linden, 1989). We write

$$
\omega=\omega_{1} t+\omega_{3} t^{3}+\ldots
$$

with expansions in even powers of $t$ for $v$ and $\theta$, and substitute the expansions into the dimensioned equations in Section 2.1; thermobaric effects are ignored since they 
were dealt with by Kay (2001). Planetary rotation first influences the vorticity at $O\left(t^{3}\right)$, and the expansion for $\omega$ to this order is found to be

$$
\omega=-G x t\left(1-\frac{1}{6} f_{z}^{2} t^{2}\right)+\frac{1}{6} G t^{3}\left(2 G x-f_{x} f_{z}\right)(h-2 z)+O\left(t^{5}\right),
$$

where

$$
G=2 \beta g \kappa^{2}
$$

is a parameter characterising the nonlinear density profile.

The first term on the right-hand side of (4.2) shows the vertical component of planetary rotation reducing the lowest-order vorticity generation, $\omega_{1} t=-G x t$, by a factor which is uniform in space and increasing with time. Remembering that $\omega$ is the $y$-component of the vorticity vector, its reduction is simply due to rotation about the $z$-axis converting some $y$-component to $x$-component: in other words, the familiar rotation of vectors which characterises inertial oscillations. To confirm this, note that the first term on the right of (4.2) looks like the expansion of $\left(\omega_{1} / f_{z}\right) \sin f_{z} t$; furthermore the $x$-component of vorticity is found to be

$$
\frac{\partial v}{\partial z}=\frac{1}{2} G f_{z} x t^{2}+f_{x}\left(z-\frac{h}{2}\right) t^{2}+O\left(t^{4}\right),
$$

in which the first term on the right looks like $\left(\omega_{1} / f_{z}\right)\left(\cos f_{z} t-1\right)$; so the vorticity components are behaving similarly to the velocity components in the inertial oscillations described by Tandon and Garrett (1994).

The values of the horizontal velocity $u$ (and its dimensionless counterpart $U$ ) at the rigid boundaries will be used in much of the analysis below to characterise the dynamics. Here, they provide useful indicators of the effect of rotation on frontogenesis. Denoting upper and lower boundaries by subscripts + and - respectively, we find by integration of (4.2) and use of the boundary conditions that

$$
u_{ \pm}=\mp \frac{1}{2} G x t+\left(-\frac{1}{18} G^{2} h^{2} x \pm \frac{1}{12} f_{z}^{2} G h x-\frac{1}{36} f_{x} f_{z} G h^{2}\right) t^{3}+O\left(t^{5}\right),
$$


in which upper (lower) signs refer everywhere to upper (lower) boundaries. The first term in the parentheses in (4.5) results from nonlinear effects - the nonlinear density profile and also the nonlinear advection terms - and indicates convergence/frontogenesis and divergence/frontolysis at the upper and lower boundaries, respectively (Kay, 1992). Noting that the Rossby number is $a=(G h)^{1 / 2} / f_{z}$, the second term in the parentheses, deriving from the vertical component of planetary rotation, is larger than the frontogenesis term if $a^{2}<3 / 2$. Whereas in a non-rotating frame $u_{+}$and $\partial u_{+} / \partial t$ are both monotonic increasing with time, in a rotating frame with $a^{2}<3 / 2$ the rate of acceleration of the upper boundary flow will initially decrease, which will obviously delay frontogenesis. Even at high Rossby numbers, when planetary rotation is slow compared with frontogenesis, a front will appear after a slightly longer time than it would in a non-rotating frame.

At very low Rossby numbers, density gradients in the upper, convergent-flow region will only have steepened a little within the first quarter-period of rotation. During the second quarter-period the convergence will decelerate due to the rotating frame; during the second half-period the flow will become divergent, causing the density gradient to slacken. However, because of the contrast between the positive feedback of convergence/frontogenesis and the negative feedback of divergence/frontolysis, the frontogenesis during the first half-period will be greater than the frontolysis during the second half-period. Thus, we may expect an overall steepening of density gradients averaged over many periods, with eventual formation of a front being likely even at low Rossby numbers (disregarding the reality that other environmental changes would be likely to disturb the system in the mean time).

The role of the horizontal component of planetary rotation is more subtle, introducing horizontal asymmetry into frontogenesis. The grouping of terms in (4.2) sets 
the effects of $f_{x}$ alongside the effects of nonlinearity, in the second term on the righthand side. To the left of the axis $x=0$, the rotation term reinforces the nonlinear effects (frontogenesis and frontolysis near the upper and lower boundaries, respectively); rotation opposes nonlinearity to the right of the axis. Thus the most intense frontogenesis will be found in the quadrant $x<0, z>h / 2$, although the effects of $f_{x}$ will only be significant within distances of order $f_{x} f_{z} / 2 G$ from the axis.

We shall henceforth ignore both thermobaricity and the horizontal component of planetary rotation; these factors were shown to be of secondary importance to frontogenesis (see remark (ii) near the end of Section 2.2), and the interaction of a nonlinear density profile with rotation about a vertical axis is sufficiently interesting without introducing further factors to obscure the results.

\subsection{Numerical solution}

The numerical method is essentially the same as that used by Kay (1992) and Grundy and Kay (2003). We integrate the unsubscripted variables along characteristics according to (2.35), except that we use the zero potential vorticity condition in the form (2.36) to reduce this to

$$
\mathrm{d} T=\frac{\mathrm{d} Z}{W}=\frac{\mathrm{d} \Omega}{-U \Omega-\Theta^{2}+a^{-2} \partial \Theta / \partial Z}=\frac{\mathrm{d} \Theta}{-U \Theta} .
$$

Cases involving inertial oscillations provide a more severe test of the numerical procedure than straightforward frontogenesis, so more characteristics were used than in the author's previous studies of non-rotating frontogenesis. Chebyshev polynomials were used to interpolate values of $\Omega$ and $\Theta$ in order to facilitate the integrations and differentiations necessary to find $U$ and $W$ (according to (2.31)) and $\partial \Theta / \partial Z$ (required in (4.6)). In some cases spurious spatial oscillations developed, which were related 
to this Chebyshev interpolation. These oscillations could be eliminated by the use of cubic B-splines (Cox, 1975) as an alternative means of interpolation; however, tests with the non-rotating case showed that splines were less able to represent the extreme behaviour of $\Omega$ and $\Theta$ near the upper boundary in the final stages of frontogenesis. This leads to inaccurate estimates of the time $T_{b}$ at which a singularity first appears, referred to henceforth as the "blow-up time". Thus, Chebyshev polynomials have been used used in most of the calculations presented below, but calculations with splines have also been performed to check that any numerical oscillations which develop do not have a significant effect on the overall frontogenesis process. A further check is made by evaluating the conserved integral $I_{0}$ from (2.37) at each time-step, and ensuring that any deviations from the initial value of unity are small.

The results for large values of Rossby number show the expected behaviour, with frontogenesis proceeding in a similar manner to the non-rotating case, but more slowly. Table I quantifies this delay, with blow-up times increasing smoothly as Rossby number decreases down to 0.956. Note the contrast with the results from geostrophic adjustment theory, which predicts no singularity for $a<\sqrt{2}$; the initial value problem shows blow-up occurring without any inertial oscillations for $a \geq 0.956$, and occurring after one or more oscillations for Rossby numbers lower than this. The sudden change in values of $T_{b}$ between $a=0.956$ and $a=0.955$ is due to the system undergoing one complete oscillation before the final frontogenesis at the smaller value of Rossby number. To illustrate the change in behaviour, we plot $\Omega_{+}$(where subscripts + and - again indicate values attained at the upper and lower boundaries, $Z=1$ and $Z=0$ respectively) against $T$ for the cases $a=0.96$ and $a=0.95$ in Figure 1. The two cases are almost indistinguishable for $T<2.0$, and their behaviours remain very similar until $T \approx 3.6$ : the growth in the negative value of $\Omega_{+}$is initially 
steady, but then accelerates rapidly. However, whereas with the larger value of $a$ the runaway growth continues until a singularity appears at $T \approx 3.927$, with $a=0.95$ there is a turning point after which $\Omega_{+}$returns rapidly to zero and soon attains a large positive value. It then returns more slowly to zero and blows up negatively at $T \approx 8.56$ in a similar manner to the earlier blow-up of the case $a=0.96$.

The behaviour of $U_{+}$for the same two values of Rossby number, shown in Figure 2 , is similar to that of $\Omega_{+}$but with much smaller magnitudes at the turning points in the case $a=0.95$. In the early stages, the rate of growth of $U_{+}$is actually slowing down a little, as predicted in Section 4.1 above for $a<(3 / 2)^{1 / 2} \approx 1.225$. Figure 2 also shows $U_{-}$, which displays oscillatory behaviour before the final approach to the singularity for both values of Rossby number. Nevertheless, it is the flow near the upper boundary that drives the formation of a singularity and thus determines the blow-up time (Grundy and Kay, 2003).

It is not possible to determine analytically the critical value of Rossby number that separates the two behaviours shown in Figure 1. However, noting that the rigid boundaries are characteristics, we may write

$$
\frac{\mathrm{d} \Omega_{+}}{\mathrm{d} T}=-U_{+} \Omega_{+}-\Theta_{+}^{2}+a^{-2}\left[\frac{\partial \Theta}{\partial Z}\right]_{+}
$$

from (4.6). In the early stages of the flow, the two nonlinear terms on the righthand side of (4.7) both give negative contributions to $\mathrm{d} \Omega_{+} / \mathrm{d} T$. The final term, which arises from rotation, gives a positive contribution. As frontogenesis proceeds, steepening density gradients are confined to an ever narrower layer near the upper boundary (Grundy and Kay, 2003), so $[\partial \Theta / \partial Z]_{+}$grows more rapidly than $\Theta_{+}$. If $a$ is sufficiently small, this growth may overcome that of the nonlinear terms, leading to a change in sign of $\mathrm{d} \Omega_{+} / \mathrm{d} T$, as seen in figure 1 for $a=0.95$. Noting from (2.29), 
(2.31) and (2.34) that

$$
\left[\frac{\partial \Theta}{\partial Z}\right]_{+}=-\Omega_{+} \Theta_{+},
$$

we see that $[\partial \Theta / \partial Z]_{+}$continues to grow until $\Omega_{+}$itself changes sign; this presumably accounts for the very steep temporal gradient of $\Omega_{+}$from the trough to the peak in Figure 1.

As the Rossby number $a$ decreases beyond 0.955, there are further discontinuous jumps in the value of blow-up time $T_{b}$ at $a \approx 0.843, a \approx 0.768$ and $a \approx 0.714$ (see Table I), and at further values of $a$ which are increasingly closely packed as $a$ decreases. These correspond to increases in the number of complete oscillations before blow-up. One curiosity is the non-monotonic variation of $T_{b}$ with $a$ : as $a$ decreases from each of the critical values at which the jumps in $T_{b}$ occur, $T_{b}$ initially decreases and only starts increasing again shortly before the next jump. This reflects the scaling of our dimensionless time $T$ with respect to a time-scale of frontogenesis rather than planetary rotation. The former scaling is required for comparisons with the non-rotating case, and is appropriate at high Rossby numbers; however, at lower Rossby numbers a dimensionless time scaled by the coriolis period,

$$
\widetilde{T}=f_{z} t=\frac{T}{a}
$$

may be more appropriate. Blow-up times $\widetilde{T}_{b}$ scaled in this way are plotted for Rossby numbers between 0.7 and 1.05 in Figure 3. Here, jumps in $\widetilde{T}_{b}$ of approximately $2 \pi$ (i.e. one coriolis period) are separated by regions in which the blow-up time is seen to remain fairly constant, indicating that blow-up always occurs at roughly the same stage of the inertial oscillation cycle.

The situation for a lower value of Rossby number, $a=0.5$, is shown in the plot of $U_{+}$and $U_{-}$in Figure 4. Inertial oscillations are seen to grow in amplitude, with the 
rate of growth accelerating; the waveforms also show increasing deviations from the sinusoidal form of a linear oscillation as time goes on, with $U_{+}$eventually displaying the same steep growth from troughs to peaks that was seen for $a=0.95$ in Figure 2 . The period of the oscillations is slightly shorter for $U_{-}$than for $U_{+}$, so that the phase difference between $U_{-}$and $U_{+}$gradually shifts from its initial value of $\pi$. Eventually (at $T \approx 34.5$ ), when this phase shift has nearly reached $\pi / 2$, there is a sudden change in behaviour as the oscillations give way to runaway growth towards a singularity; but we have been unable to determine analytically whether the phase shift does indeed provide a criterion for the transition between oscillatory and blow-up behaviours.

It can be shown that the rotation term in (4.6) has no effect on the structure of the flow on the approach to the singularity, to the asymptotic order calculated by Grundy and Kay (2003) for non-rotating flow, so this final stage of frontogenesis is unaffected by rotation and will not be discussed further here. It has not been possible to prove rigorously that a singularity does actually appear in finite time for any finite value of Rossby number: the first step of the Jacqmin (1991) proof of blow-up is invalidated by the presence of a rotation term in the vorticity equation. Nevertheless, our numerical evidence suggests that a finite-time singularity does probably occur eventually for any non-zero value of $a$, and this is reasonable since there is an infinite supply of potential energy. In any case, the final blow-up is the least physically realistic part of the dynamics, whereas the inertial oscillations that precede it are of considerable physical relevance. We now proceed with a detailed analysis of these oscillations for low Rossby number. 


\subsection{Analysis of the oscillations}

Figure 4 clearly indicates that at low values of Rossby number there are two dynamical processes, operating on different time-scales: the period of the inertial oscillations is a fast time-scale, while frontogenesis, seen in the growth in amplitude of the oscillations, operates on a slow time-scale (Blumen, 1997). This motivates an analysis by the method of multiple scales (e.g. Nayfeh (1973)) to obtain $W$ and $\Theta$ as expansions in powers of Rossby number; details are given in the Appendix.

Recalling the definitions of the similarity variables (equations (2.17) - (2.21)), we note that $U, \Theta$ and $V$ are proportional to the divergence of the horizontal flow, the horizontal temperature gradient and the vertical component of relative vorticity, respectively. It is instructive to examine the values of these variables at the upper and lower boundaries: from (A.25) and (A.26), using (A.3) and writing in terms of the original dimensionless time $T$, we obtain

$$
\begin{aligned}
U_{ \pm}=a(\mp & \left.\frac{1}{2} \sin \frac{T}{a}\right)+a^{2}\left(\frac{T}{24} \cos \frac{T}{a}\right)+a^{3}\left(\mp \frac{T^{2}}{192} \sin \frac{T}{a}-\frac{1}{8} \sin \frac{T}{a}+\frac{1}{24} \sin \frac{2 T}{a}\right) \\
& +a^{4}\left(\frac{T^{3}}{6912} \cos \frac{T}{a} \pm \frac{T}{48} \cos \frac{T}{a} \mp \frac{T}{48} \cos \frac{2 T}{a}\right)+O\left(a^{5}\right) \\
\Theta_{ \pm}=1+ & a^{2}\left( \pm \frac{1}{2}\left(1-\cos \frac{T}{a}\right)\right)+a^{3}\left(-\frac{T}{24} \sin \frac{T}{a}\right) \\
& +a^{4}\left(\mp \frac{T^{2}}{192} \cos \frac{T}{a}+\frac{1}{3}-\frac{5}{12} \cos \frac{T}{a}+\frac{1}{12} \cos \frac{2 T}{a}\right) \\
& +a^{5}\left(-\frac{T^{3}}{6912} \sin \frac{T}{a} \mp \frac{T}{32} \sin \frac{T}{a} \pm \frac{T}{48} \sin \frac{2 T}{a}\right)+O\left(a^{6}\right)
\end{aligned}
$$

Formulae for $V_{ \pm}$are then obtained from (4.11) using the zero potential vorticity condition,

$$
V_{ \pm}=\left(\Theta_{ \pm}-1\right) / a
$$


The above asymptotic expansions may be compared with the numerical results shown in Figures 4 and 5 for $a=0.5$, and have also been verified to high precision by a numerical solution for $a=0.1$ (not shown). From them we may extract details of the time-varying amplitude, period and shape of the inertial oscillations, as explained below.

We may write (4.10) in the form

$$
U_{ \pm}=\widehat{U}_{ \pm} \sin \left(\frac{T}{a}+\phi_{ \pm}\right)+\text {second harmonic terms }
$$

where

$$
\widehat{U}_{ \pm}=\mp \frac{1}{2} a-\frac{1}{8} a^{3} \mp \frac{1}{72} a^{3} T^{2}+O\left(a^{5}\right)
$$

and

$$
\tan \phi_{ \pm}=\mp \frac{1}{12} a T-\frac{1}{48} a^{3} T \pm \frac{1}{1728} a^{3} T^{3}+O\left(a^{5}\right) .
$$

The amplitude of linear oscillations is $a / 2$ (or, in dimensioned variables, the divergence $\partial u / \partial x$ has amplitude $\kappa^{2} \beta g h / f_{z}$ ), and this is perturbed in two ways. Firstly, the amplitude is increased (decreased) by an amount $a^{3} / 8$ at the upper (lower) boundary; this is a lasting effect of the convergence/divergence near the upper/lower boundary during the first half-period. Secondly, the amplitude grows quadratically over time, as is seen in figure 4; this is the net effect of the convergence/frontogenesis and the divergence/frontolysis within each period, which do not exactly cancel out because the former process is self-reinforcing while the latter is self-weakening.

The angular frequency of linear oscillations is $1 / a$, or $f_{z}$ in dimensioned variables. From (4.15) we obtain

$$
\sin \left(\frac{T}{a}+\phi_{ \pm}\right)=\sin \left\{T\left(\frac{1}{a} \mp \frac{a}{12}-\frac{a^{3}}{48} \pm \frac{a^{3}}{1296} T^{2}+O\left(a^{5}\right)\right)\right\},
$$

i.e. the frequency is less (greater) than that of linear oscillations at the upper (lower) boundary, so that the period is greater where the amplitude is greater. In fact, even for 
Rossby number as high as $a=0.95$ the periods $2 \pi a\left(1 \pm a^{2} / 12\right) \approx 5.97 \pm 0.45$ predicted to $O\left(a^{3}\right)$ by $(4.16)$ are well approximated by the durations of the single complete oscillation of $U_{+}$and $U_{-}$in Figure 2, which are 6.52 and 5.49 respectively. Higherorder effects are that the perturbation of frequency from its linear value is greater at the upper boundary and less at the lower boundary, and that this perturbation gradually reduces over time.

The second harmonic terms in (4.10) may be re-written as oscillations at twice the first-order perturbed frequency:

$$
\begin{aligned}
\frac{a^{3}}{24} \sin & \frac{2 T}{a} \mp \frac{a^{4}}{48} T \cos \frac{2 T}{a} \\
= & \frac{a^{3}}{24} \sin \left\{2 T\left(\frac{1}{a} \mp \frac{a}{12}\right)\right\} \mp \frac{a^{4}}{72} T \cos \left\{2 T\left(\frac{1}{a} \mp \frac{a}{12}\right)\right\}+O\left(a^{5}\right) .
\end{aligned}
$$

The sine term in (4.17) produces a distortion in the wave form, of the sort which is seen in an extreme form for $U_{ \pm}$in Figure 2 and is also discernible at larger times in Figure 4, where the rise from the trough to the peak is steeper than the descent back to the trough. The cosine term affects amplitudes, since $\cos 2 t$ has a minimum coinciding with every maximum and minimum of $\sin t$; thus its overall effect is to produce an asymmetry between amplitudes of peaks and troughs in the oscillations, and also to shift the origins of the quadratic growth in amplitude to $T=-a$ (for peaks of $U_{+}$and troughs of $U_{-}$) or to $T=a$ (for peaks of $U_{-}$and troughs of $U_{+}$).

The oscillations of $\Theta_{ \pm}$seen in Figure 5 show similar behaviour in terms of amplitude, period and waveform to those of $U_{ \pm}$, as evidenced by the similarities between the expansions (4.10) and (4.11). Where $\Theta_{ \pm}$differs significantly from $U_{ \pm}$is in its mean value over a period,

$$
\overline{\Theta_{ \pm}}=1 \pm \frac{1}{2} a^{2}+\frac{4}{3} a^{4}+O\left(a^{6}\right)
$$


where the overbar represents the mean over an oscillation period of length $2 \pi a$. The $O\left(a^{2}\right)$ perturbation of the period-averaged $\Theta_{ \pm}$from the initial condition $\Theta=1$ demonstrates the lasting effect of frontogenesis (frontolysis) at the upper (lower) boundary in the first half-period. Figure 5 also shows a quadratic increase in the period-averaged value of $\Theta$ over the frontogenesis time scale, which is due to terms at $O\left(a^{6} T^{2}\right)$ in (4.11), or $O\left(a^{4} S^{2}\right)$ in (A.26). These terms have not been calculated, but using (A.13) we may obtain the vertical mean temperature gradient as

$$
\begin{aligned}
\int_{0}^{1} \Theta d Z=1+ & a^{4}\left\{\frac{1}{24}(3-4 \cos \tau+\cos 2 \tau)+\frac{S^{2}}{2880}(3-5 \cos \tau+2 \cos 2 \tau)+O\left(S^{4}\right)\right\} \\
+ & O\left(a^{6}\right),
\end{aligned}
$$

so that the period- and spatially-averaged temperature gradient is perturbed from its initial value by a constant fraction $a^{4} / 8$ and a quadratically growing fraction $a^{6} T^{2} / 960$, to lowest order.

From (4.12) the behaviour of the vertical relative vorticity will be similar to that of the temperature gradient, and we again consider the period- and spatially-averaged value of this parameter,

$$
\int_{0}^{1} \bar{V} d Z=\left\{\frac{1}{8} a^{3}+O\left(a^{5}\right)\right\}+\left\{\frac{1}{960} a^{5}+O\left(a^{7}\right)\right\} T^{2}+O\left(T^{4}\right) .
$$

There is a net positive (cyclonic) vorticity, resulting from the contrast between the positive feedback of the frontogenesis/convergence process and the negative feedback of the frontolysis/divergence process: a convergent flow will be turned by the coriolis force to produce a cyclonically rotating flow, which will be stronger than the anticylonic flow in the divergent region. The averaged cyclonic vorticity consists of a constant component $(\beta g h)^{2} \kappa^{4} / 2 f_{z}^{3}$ (in dimensioned variables) which is the lasting legacy of the flow during the first half-period, together with a quadratically growing 
component $(\beta g h)^{4} \kappa^{8} t^{2} / 60 f_{z}^{5}$ reflecting the net effect of convergence and divergence during subsequent oscillations.

The flow will become unstable if the vertical density gradient $\partial \rho / \partial z$ becomes positive or if a stable stratification is insufficient to overcome the destabilizing effect of shear. In our dimensionless variables the former condition is

$$
\Theta \frac{\partial \Theta}{\partial Z}<0
$$

while the latter situation occurs if the gradient Richardson number,

$$
\mathrm{Ri}=\frac{-\frac{g}{\rho_{m}} \frac{\partial \rho}{\partial z}}{\left(\frac{\partial u}{\partial z}\right)^{2}+\left(\frac{\partial v}{\partial z}\right)^{2}}=\frac{\Theta \frac{\partial \Theta}{\partial Z}}{\left(\frac{\partial U}{\partial Z}\right)^{2}+\left(\frac{\partial V}{\partial Z}\right)^{2}}
$$

falls below 1/4. For linear inertial oscillations starting from initial conditions of a fluid at rest with a horizontal density gradient, a value $\mathrm{Ri}=1 / 2$ is expected (Tandon and Garrett, 1994). In the present case, we find

$$
\Theta \frac{\partial \Theta}{\partial Z}=a^{2}\left\{(1-\cos \tau)-\frac{S}{4}(2 Z-1) \sin \tau-\frac{S^{2}}{96} \cos \tau+O\left(S^{3}\right)\right\}+O\left(a^{4}\right)
$$

and

$$
\left(\frac{\partial U}{\partial Z}\right)^{2}+\left(\frac{\partial V}{\partial Z}\right)^{2}=\frac{1}{2} \Theta \frac{\partial \Theta}{\partial Z}+a^{2}\left\{\frac{S^{2}}{48}\left(12 Z^{2}-12 Z+4\right)+O\left(S^{4}\right)\right\}+O\left(a^{4}\right)
$$

so that $\mathrm{Ri} \sim 1 / 2$, with corrections at $O\left(a^{2}\right)$ and $O\left(a^{2} T^{2}\right)$, provided that $(1-\cos \tau)$ and $\sin \tau$ are not small. However, around $\tau=2 N \pi(N=1,2,3, \ldots)$, i.e. once during each oscillation period, there is an interval when the flow becomes statically unstable according to criterion (4.21). The duration of this unstable phase is at most a fraction $N a^{2} / \sqrt{3}$ of the oscillation period (depending on vertical position $Z$ ), and so increases with the period number $N$ in which it occurs. Furthermore, the duration of KelvinHelmholtz instability $(\mathrm{Ri}<1 / 4)$ will be longer than that of static instability. The 
occurrence of static instability is verified in Figure 5, in which phases of increasing duration where $\Theta_{+}<\Theta_{-}$appear in successive oscillation periods.

\section{Conclusions}

Following the seminal work of $\mathrm{Ou}$ (1984), frontogenesis in rotating systems confined between rigid horizontal boundaries has been investigated in a series of papers by Blumen and his collaborators. Although BL and BW find both inertial oscillations (superposed on a geostrophic flow) and frontogenesis, they do not pursue the full solution of the vorticity equation for the flow in the vertical plane containing the temperature gradient vector; hence they do not determine the development of the oscillations due to nonlinear interactions over many periods. The price paid in the present work for rectifying this is that we have used rather unrealistic initial conditions: although the quadratic density profile has a sound basis in the equation of state of fresh water, its infinite extent, which permits a mathematically elegant similarity solution, is unphysical.

The most interesting case analysed above is that of low Rossby number, when the time scale for frontogenesis is long compared with the period of inertial oscillations. Solutions were obtained in the form of asymptotic expansions in the Rossby number as a small parameter. The oscillations are seen to grow over time: although both frontogenesis and frontolysis occur within each oscillation period, frontogenesis and horizontal flow convergence form a positive feedback loop while there is negative feedback between frontolysis and flow divergence; thus there is a net increase in temperature gradients from one period to the next. Similarly, whereas at any instant frontogenesis at one boundary may be accompanied by frontolysis at the op- 
posite boundary, the spatially averaged temperature gradient increases and there is an associated net cyclogenesis.

Blow-up, which can be interpreted as a proxy for the appearance of a sharp front, appears to occur eventually for all positive values of Rossby number, but it has not been possible to prove this rigorously. In its final stages, the flow takes the same form as in the non-rotating case, as described in detail by Grundy and Kay (2003). However, there remain some questions relating to earlier stages of the approach to blow-up, which cannot be answered by asymptotic solutions. The blow-up time is not a smooth function of Rossby number (see Table I), and it is not clear what determines critical values of Rossby number at which the number of oscillations before blow-up changes. There appears to be a sudden transition from oscillatory behaviour to a rapid approach to blow-up, which may be related to the desynchronisation of the oscillations across the flow domain: the nonlinear interactions lengthen the period of oscillation at one boundary and shorten it at the other. However, we have not been able to analyse the exact dynamical process by which the transition to blow-up occurs.

In the similarity solution we included some factors not kept in the subsequent numerical and asymptotic analysis. Thermobaricity is specific to deep water bodies, but the horizontal component of planetary rotation is more generally applicable. Nevertheless, we showed that both of these factors can only modify the details of the flow, and cannot affect the process of growing inertial oscillations leading to blow-up.

We have already commented on the unphysical nature of the unbounded density variations in our model problem. However, this should not obscure the fact that inertial oscillations of growing amplitude may occur whenever there is a nonlinear horizontal density variation. On the other hand, the discovery of instability occurring 
once in each oscillation period may lend some support to BW's decision to only analyse the flow for one oscillation period. Even if the instability does not prevent oscillations continuing, it does suggest that one should at least take account of turbulent mixing in further work on this phenomenon.

\section{Appendix: Multiple-scales analysis for inertial os- cillations at low Rossby number}

The equations to be solved are given in Section 2.2, but we restate them here for convenience, using the zero potential vorticity condition (2.36) to eliminate $V$ :

$$
\begin{aligned}
& \frac{\partial \Omega}{\partial T}=-U \Omega-W \frac{\partial \Omega}{\partial Z}+\frac{1}{a^{2}} \frac{\partial \Theta}{\partial Z}-\Theta^{2} \\
& \frac{\partial \Theta}{\partial T}=-U \Theta-W \frac{\partial \Theta}{\partial Z}
\end{aligned}
$$

where

$$
\begin{gathered}
\Omega=\frac{\partial U}{\partial Z}=-\frac{\partial^{2} W}{\partial Z^{2}} \\
W=U=\Omega=0, \quad \Theta=1 \quad \text { at } \quad t=0 ; \\
W=0 \text { at } Z=0 \text { and at } Z=1 .
\end{gathered}
$$

The conservation law

$$
\int_{0}^{1} \frac{1}{\Theta} \mathrm{d} Z=1
$$

may be derived from the above equations.

We define a fast time

$$
\tau=\frac{T}{a}
$$


and a slow time

$$
S=a T,
$$

which are treated as separate independent variables; thus

$$
\frac{\partial}{\partial T}=\frac{1}{a} \frac{\partial}{\partial \tau}+a \frac{\partial}{\partial S} .
$$

The dependent variables are expanded as

$$
\Omega=a \Omega_{1}+a^{3} \Omega_{3}+\ldots
$$

(and similarly for $U$ and $W$ ) and

$$
\Theta=1+a^{2} \Theta_{2}+a^{4} \Theta_{4}+\ldots,
$$

where the leading-order term in (A.11) is determined by the initial condition (A.4). Expanding the conservation law (A.6) yields

$$
\begin{aligned}
& \int_{0}^{1} \Theta_{2} d Z=0 \\
& \int_{0}^{1} \Theta_{4} d Z=\int_{0}^{1} \Theta_{2}^{2} d Z
\end{aligned}
$$

as conditions on terms in the expansion (A.11).

On substituting the expansions (A.10) and (A.11) into (A.1) and (A.2), then extracting $O(1)$ terms, we obtain

$$
\begin{aligned}
& \frac{\partial \Omega_{1}}{\partial \tau}=-1+\frac{\partial \Theta_{2}}{\partial Z} \\
& \frac{\partial \Theta_{2}}{\partial \tau}=-U_{1} .
\end{aligned}
$$

Using (A.3) to express these equations in terms of $W$ and $\Theta$, and applying boundary conditions (A.5) and (A.12) and initial conditions (A.4), we obtain the solution

$$
\begin{aligned}
& W_{1}=A \sin \tau+B \cos \tau \\
& \Theta_{2}=Z-\frac{1}{2}-\frac{\partial A}{\partial Z} \cos \tau+\frac{\partial B}{\partial Z} \sin \tau
\end{aligned}
$$


where the amplitude coefficients $A(Z, S)$ and $B(Z, S)$ must satisfy

$$
\begin{aligned}
& A(Z, 0)=\frac{1}{2}\left(Z^{2}-Z\right) \quad \text { and } \quad B(Z, 0)=0 \\
& A(0, S)=A(1, S)=B(0, S)=B(1, S)=0 .
\end{aligned}
$$

Equations (A.16) and (A.17) describe inertial oscillations similar to those found by BL, except that BL's amplitude coefficients did not admit any variation with the slow time $S$. To fully determine $A(Z, S)$ and $B(Z, S)$, we proceed to $O\left(a^{2}\right)$ in $(\mathrm{A} .1)$ and (A.2), obtaining

$$
\begin{aligned}
\frac{\partial \Omega_{3}}{\partial \tau} & =-\frac{\partial \Omega_{1}}{\partial S}-U_{1} \Omega_{1}-W_{1} \frac{\partial \Omega_{1}}{\partial Z}+\frac{\partial \Theta_{4}}{\partial Z}-2 \Theta_{2} \\
\frac{\partial \Theta_{4}}{\partial \tau} & =-\frac{\partial \Theta_{2}}{\partial S}-U_{1} \Theta_{2}-U_{3}-W_{1} \frac{\partial \Theta_{2}}{\partial Z}
\end{aligned}
$$

Using (A.3), we eliminate $\Theta_{4}$ and substitute the leading-order results (A.16) and (A.17) to yield

$$
\begin{aligned}
\frac{\partial^{2} \Omega_{3}}{\partial \tau^{2}}+\Omega_{3} & =\left\{2 \frac{\partial^{3} A}{\partial S \partial Z^{2}}+\frac{\partial^{2} B}{\partial Z^{2}}\left(Z-\frac{1}{2}\right)-2 \frac{\partial B}{\partial Z}\right\} \cos \tau \\
& +\left\{-2 \frac{\partial^{3} B}{\partial S \partial Z^{2}}+\frac{\partial^{2} A}{\partial Z^{2}}\left(Z-\frac{1}{2}\right)-2 \frac{\partial A}{\partial Z}\right\} \sin \tau \\
& +\frac{1}{2}\left\{\frac{\partial^{3} A}{\partial Z^{3}} B+\frac{\partial^{2} A}{\partial Z^{2}} \frac{\partial B}{\partial Z}-\frac{\partial A}{\partial Z} \frac{\partial^{2} B}{\partial Z^{2}}-A \frac{\partial^{3} B}{\partial Z^{3}}\right\} \\
& +\frac{3}{2}\left\{\frac{\partial^{3} A}{\partial Z^{3}} B-\frac{\partial^{2} A}{\partial Z^{2}} \frac{\partial B}{\partial Z}-\frac{\partial A}{\partial Z} \frac{\partial^{2} B}{\partial Z^{2}}+A \frac{\partial^{3} B}{\partial Z^{3}}\right\} \cos 2 \tau \\
& +\frac{3}{2}\left\{A \frac{\partial^{3} A}{\partial Z^{3}}-\frac{\partial A}{\partial Z} \frac{\partial^{2} A}{\partial Z^{2}}-B \frac{\partial^{3} B}{\partial Z^{3}}+\frac{\partial B}{\partial Z} \frac{\partial^{2} B}{\partial Z^{2}}\right\} \sin 2 \tau
\end{aligned}
$$

Forcing at the fundamental frequency would produce resonance in $\Omega_{3}$, causing the second term in the expansion (A.10) to eventually become larger than the first term, 
contradicting the asymptoticness of the expansion. Thus the coefficients of $\cos \tau$ and $\sin \tau$ in (A.22) must be set to zero, yielding a pair of coupled partial differential equations for $A(Z, S)$ and $B(Z, S)$, to be solved subject to the conditions (A.18) and (A.19). In line with the multiple time-scales philosophy, we solve these equations by expanding in powers of $S$; the number of oscillation periods for which such a solution will remain valid is of order $\tau / S=a^{-2}$. The result of this calculation is

$$
\begin{aligned}
& A=\frac{1}{2}\left(Z^{2}-Z\right)\left(1+\frac{1}{96} S^{2}\right)+O\left(S^{4}\right) \\
& B=-\frac{1}{24}\left(2 Z^{3}-3 Z^{2}+Z\right)\left(S+\frac{1}{288} S^{3}\right)+O\left(S^{5}\right),
\end{aligned}
$$

in which the small coefficients of $S^{2}$ and $S^{3}$ suggest that the expansion may remain quite accurate for larger values of $S$ than originally expected.

We can now return to (A.22), substituting our expansions for $A$ and $B$ into the non-oscillatory and second-harmonic forcing terms, which have arisen from nonlinear terms in the original system of equations. The solution of (A.22) consists of a particular integral representing the response to these forcings, together with a complementary function quantifying how nonlinear effects perturb the fundamental oscillation at $O\left(a^{2}\right)$. We only keep terms to $O(S)$ in this $O\left(a^{2}\right)$ solution, consistent with the expansions to $O\left(S^{3}\right)$ in the amplitudes of the leading-order $\left(O\left(a^{0}\right)\right)$ oscillations. Integrating $\Omega_{3}$ twice with respect to $Z$ to obtain $W_{3}$, and using (A.21) to find $\Theta_{4}$, we finally combine these results with those at leading order to obtain the expansions 


$$
\begin{aligned}
& W=a\left\{\frac{1}{2}\left(Z^{2}-Z\right) \sin \tau-\frac{S}{24}\left(2 Z^{3}-3 Z^{2}+Z\right) \cos \tau\right. \\
& \left.+\frac{S^{2}}{192}\left(Z^{2}-Z\right) \sin \tau-\frac{S^{3}}{6912}\left(2 Z^{3}-3 Z^{2}+Z\right) \cos \tau+O\left(S^{4}\right)\right\} \\
& +a^{3}\left\{\frac{1}{24}\left(2 Z^{3}-3 Z^{2}+Z\right)(3 \sin \tau-\sin 2 \tau)\right. \\
& \left.+\frac{S}{48}\left(\left(Z^{4}-2 Z^{3}+Z^{2}\right)(\cos 2 \tau-1)+\left(Z^{2}-Z\right)(\cos 2 \tau-\cos \tau)\right)+O\left(S^{2}\right)\right\} \\
& +O\left(a^{5}\right) \\
& \Theta=1+a^{2}\left\{\frac{1}{2}(2 Z-1)(1-\cos \tau)-\frac{S}{24}\left(6 Z^{2}-6 Z+1\right) \sin \tau\right. \\
& \left.-\frac{S^{2}}{192}(2 Z-1) \cos \tau-\frac{S^{3}}{6912}\left(6 Z^{2}-6 Z+1\right) \sin \tau+O\left(S^{4}\right)\right\} \\
& +a^{4}\left\{\frac{1}{24}\left(\left(6 Z^{2}-6 Z+1\right)(5-6 \cos \tau+\cos 2 \tau)+(3-4 \cos \tau+\cos 2 \tau)\right)\right. \\
& +\frac{S}{96}\left(\left(2 Z^{3}-3 Z^{2}+Z\right)(4 \sin 2 \tau-8 \sin \tau)+(2 Z-1)(2 \sin 2 \tau-3 \sin \tau)\right) \\
& \left.+O\left(S^{2}\right)\right\}+O\left(a^{6}\right)
\end{aligned}
$$

in which terms are arranged so that each of the polynomials in $Z$ that appears is either zero at both boundaries or else has zero integral over $[0,1]$.

\section{References}

Amin, N. \& Riley, N. "Horizontal free convection." Proc. Roy. Soc. Lond. A 427, 371 $-384(1990)$. 
Blumen, W. "A model of inertial oscillations with deformation frontogenesis." $J$. Atmos. Sci. 54, $2681-2692$ (1997).

Blumen, W. "Inertial oscillations and frontogenesis in a zero potential vorticity model." J. Phys. Oceanogr. 30, 31 - 39 (2000).

Blumen, W. \& Lundquist, J.K. "Spin-up and spin-down in rotating fluid exhibiting inertial oscillations and frontogenesis." Dyn. Atmos. Oceans 33, 219 - 237 (2001).

Blumen, W. \& Williams, R.T. "Unbalanced frontogenesis. Part 1: Zero potential vorticity." J. Atmos. Sci. 58, 2180 - 2195 (2001).

Blumen, W. \& Wu, R. "Geostrophic adjustment: frontogenesis and energy conversion." J. Phys. Oceanogr. 25, 428 - 438 (1995).

Cox, M.G. "An algorithm for spline interpolation." J. Inst. Maths. Applics. 15, 95 $108(1975)$.

Farmer, D.M. \& Carmack, E.C. "Wind mixing and restratification in a lake near the temperature of maximum density." J. Phys. Oceanogr. 11, 1516 - 1533 (1981).

Grundy, R.E. \& Kay, A. "The asymptotics of blow-up in inviscid Boussinesq flow and related systems." IMA J. Appl. Math. 68, $47-81$ (2003).

Jacqmin, D. "Frontogenesis driven by horizontally quadratic distributions of density." J. Fluid Mech. 228, $1-24$ (1991).

Kay, A. "Frontogenesis in gravity-driven flows with non-uniform density gradients." J. Fluid Mech. 235, 529 - 556 (1992).

Kay, A. "Thermobaric flow." Dyn. Atmos. Oceans 34, 263 - 289 (2001). 
Nayfeh, A.H., Perturbation Methods. Wiley, New York (1973).

Ou, H.W. "Geostrophic adjustment: a mechanism for frontogenesis." J. Phys. Oceanogr. 14, $994-1000$ (1984).

Proudman, I. \& Johnson, K. "Boundary-layer growth near a rear stagnation point." J. Fluid Mech. 12, 161-168 (1962).

Rhines, P.B. "Mixing and large-scale ocean dynamics." In: Small Scale Turbulence and Mixing in the Ocean (Eds. J.C.J. Nihoul \& B.M. Jamart) pp. 263 - 284, Elsevier (1988).

Sander, J., Wolf-Gladrow, D. \& Olbers, D. "Numerical studies of open ocean deep convection." J. Geophys. Res. 100, 20579 - 20600 (1995).

Simpson, J.E. \& Linden, P.F. "Frontogenesis in a fluid with horizontal density gradients." J. Fluid Mech. 202, 1 - 16 (1989).

Tandon, A. \& Garrett, C. "Mixed layer restratification due to a horizontal density gradient." J. Phys. Oceanogr. 24, 1419 - 1424 (1994). 


\begin{tabular}{|l|l|l||l|l|l|}
\hline \multicolumn{1}{|c|}{$a$} & \multicolumn{1}{c|}{$T_{b}$} & $n$ & \multicolumn{1}{c|}{$T_{b}$} & $n$ \\
\hline$\infty$ & 2.278237 & 0 & 0.842 & 13.2 & 2 \\
5.0 & 2.29435 & 0 & 0.84 & 13.15 & 2 \\
2.0 & 2.38970 & 0 & 0.8 & 12.705 & 2 \\
1.5 & 2.49996 & 0 & 0.78 & 12.625 & 2 \\
1.05 & 2.97508 & 0 & 0.77 & 12.777 & 2 \\
1.0 & 3.19232 & 0 & 0.769 & 12.893 & 2 \\
0.975 & 3.40230 & 0 & 0.768 & 17.07 & 3 \\
0.96 & 3.6850 & 0 & 0.765 & 17.008 & 3 \\
0.957 & 3.8292 & 0 & 0.75 & 16.708 & 3 \\
0.956 & 3.927 & 0 & 0.72 & 16.348 & 3 \\
0.955 & 8.6 & 1 & 0.715 & 16.469 & 3 \\
0.954 & 8.6 & 1 & 0.714 & 20.516 & 4 \\
0.95 & 8.56 & 1 & 0.71 & 20.403 & 4 \\
0.9 & 8.363 & 1 & 0.7 & 20.145 & 4 \\
0.85 & 8.4986 & 1 & 0.5 & 36.85 & 11 \\
0.845 & 8.6461 & 1 & & & \\
\hline
\end{tabular}

Table I: Blow-up time $T_{b}$ as a function of Rossby number $a$, with the number $n$ of complete oscillations of $U_{+}$before blow-up. Confidence in the accuracy of $T_{b}$ values is indicated by the number of significant figures quoted; note that errors in the numerical integration are particularly severe for Rossby numbers just below critical values at which $n$ changes. 


\section{Figure Captions}

Figure 1: Dimensionless upper-boundary vorticity gradient $\Omega_{+}$vs. dimensionless time $T$, for different values of Rossby number $a$. Solid curve: $a=0.96$. Dotted curve: $a=0.95$.

Figure 2: Dimensionless horizontal divergence $U$ at upper and lower boundaries vs. dimensionless time $T$, for different values of Rossby number $a$. Solid curve: $U_{+}$ for $a=0.96$. Dashed curve: $U_{-}$for $a=0.96$. Dotted curve: $U_{+}$for $a=0.95$. Dash-dotted curve: $U_{-}$for $a=0.95$.

Figure 3: Blow-up time $\widetilde{T_{b}}$ (scaled by the coriolis period) vs. Rossby number $a$. All cases for which numerical solutions have been obtained with $0.7 \leq a \leq 1.05$ are plotted.

Figure 4: Dimensionless horizontal divergence $U$ at upper and lower boundaries vs. dimensionless time $T$, for $a=0.5$. Solid curve: $U_{+}$. Dashed curve: $U_{-}$.

Figure 5: Dimensionless temperature gradient $\Theta$ at upper and lower boundaries vs. dimensionless time $T$, for $a=0.5$. Solid curve: $\Theta_{+}$. Dashed curve: $\Theta_{-}$. 
A. Kay: Figure 1

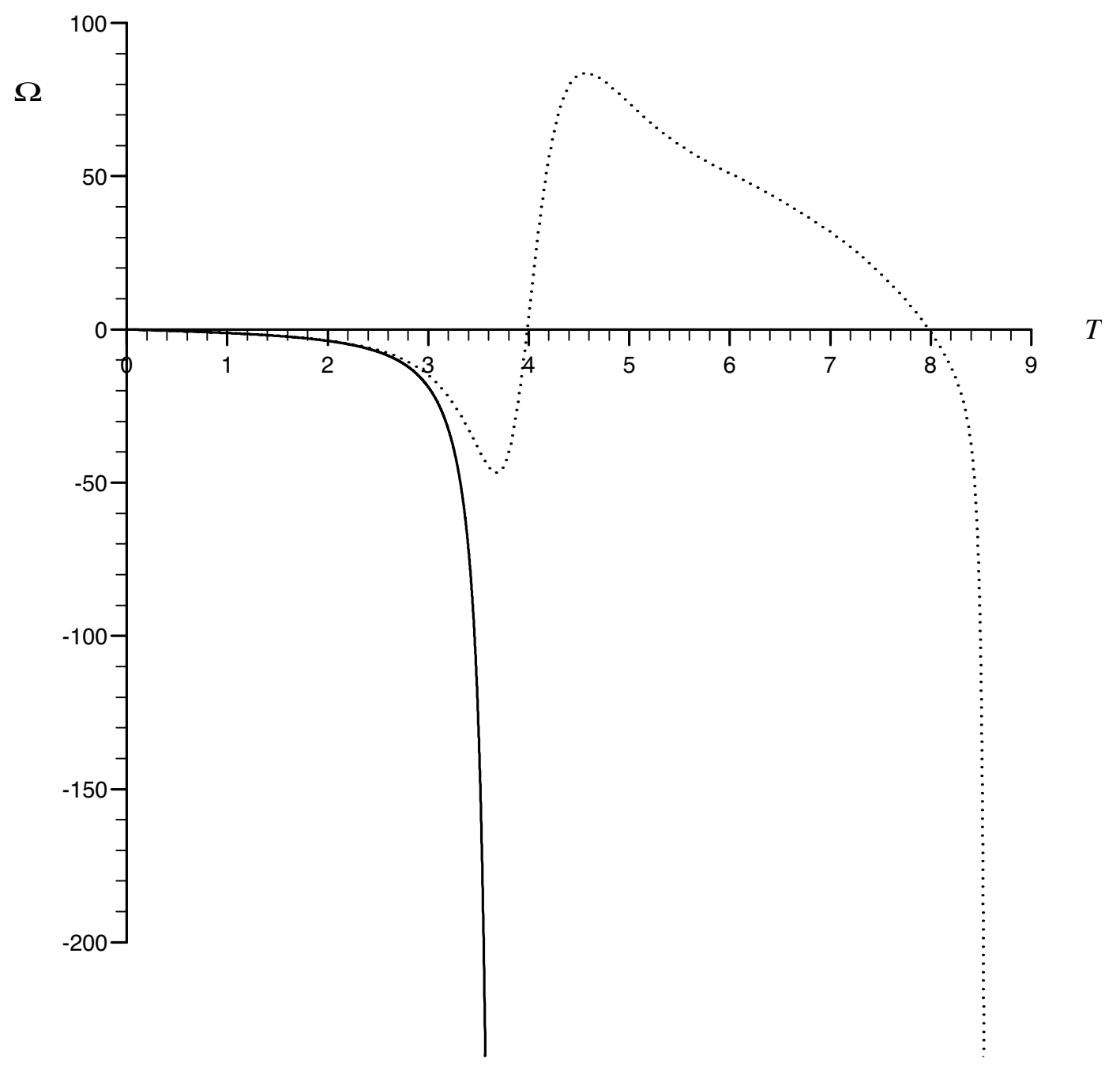

Figure 1: 
A. Kay: Figure 2

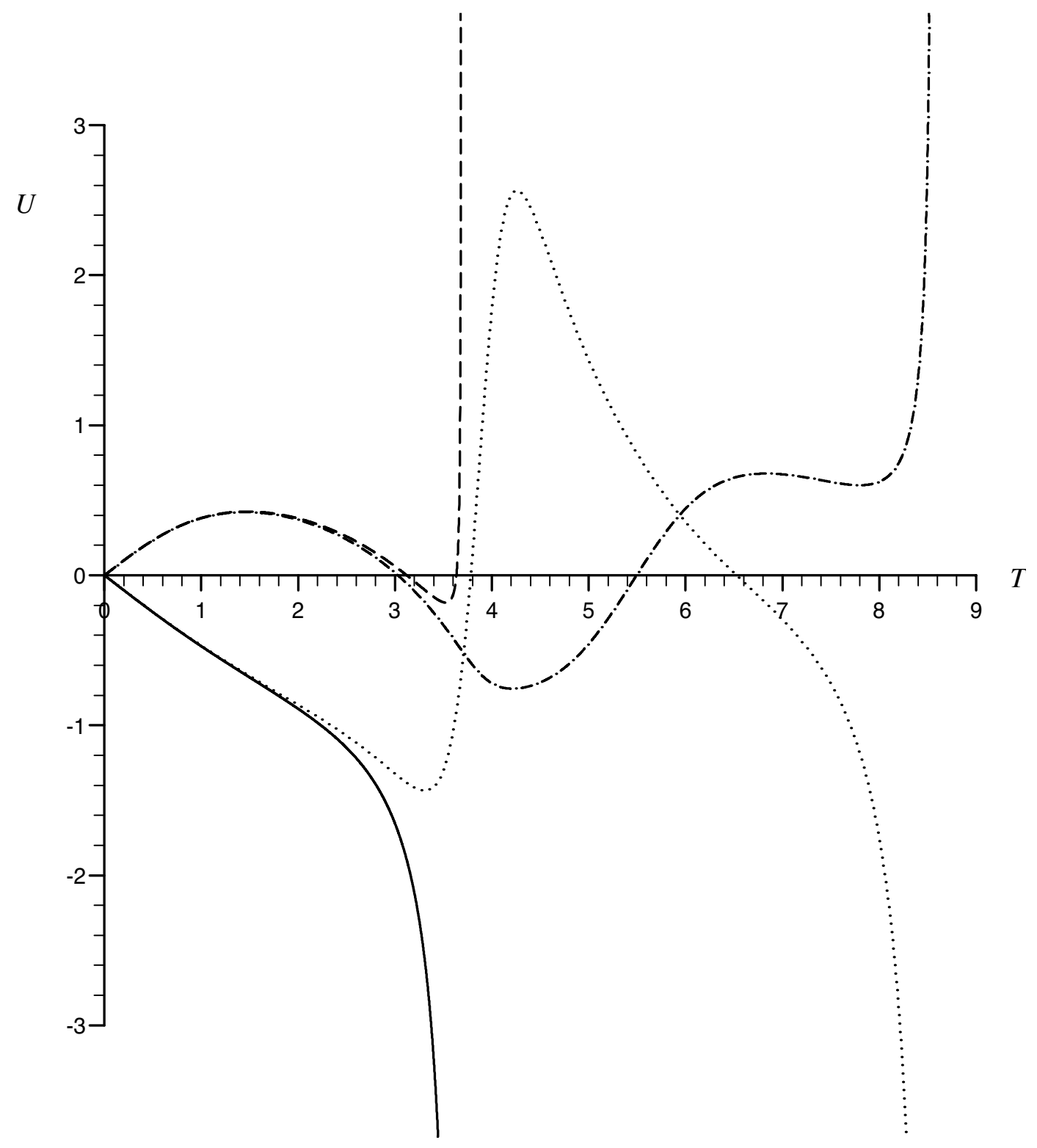

Figure 2: 
A.Kay: Figure 3

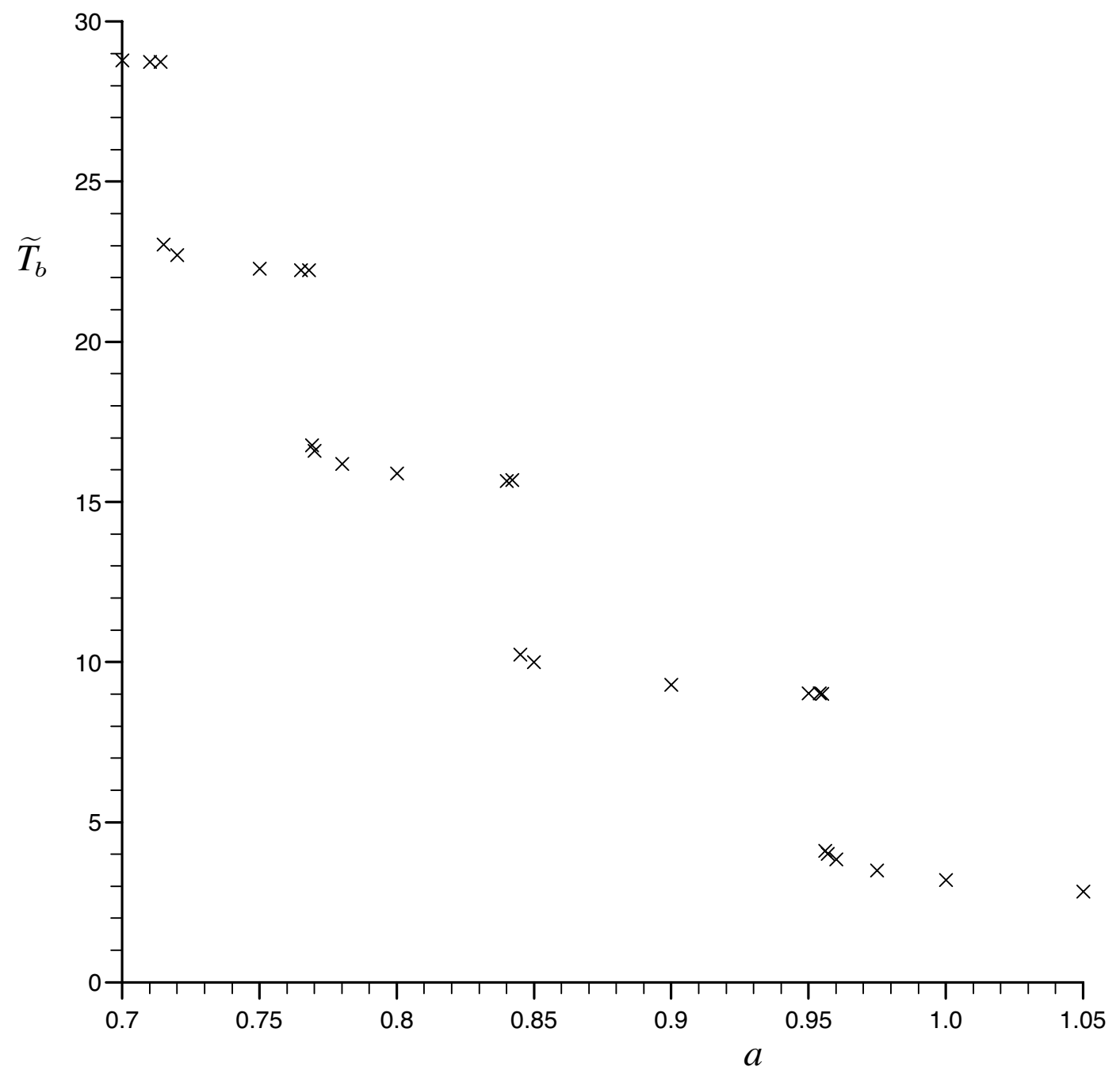

Figure 3: 


\section{A.Kay: Figure 4}

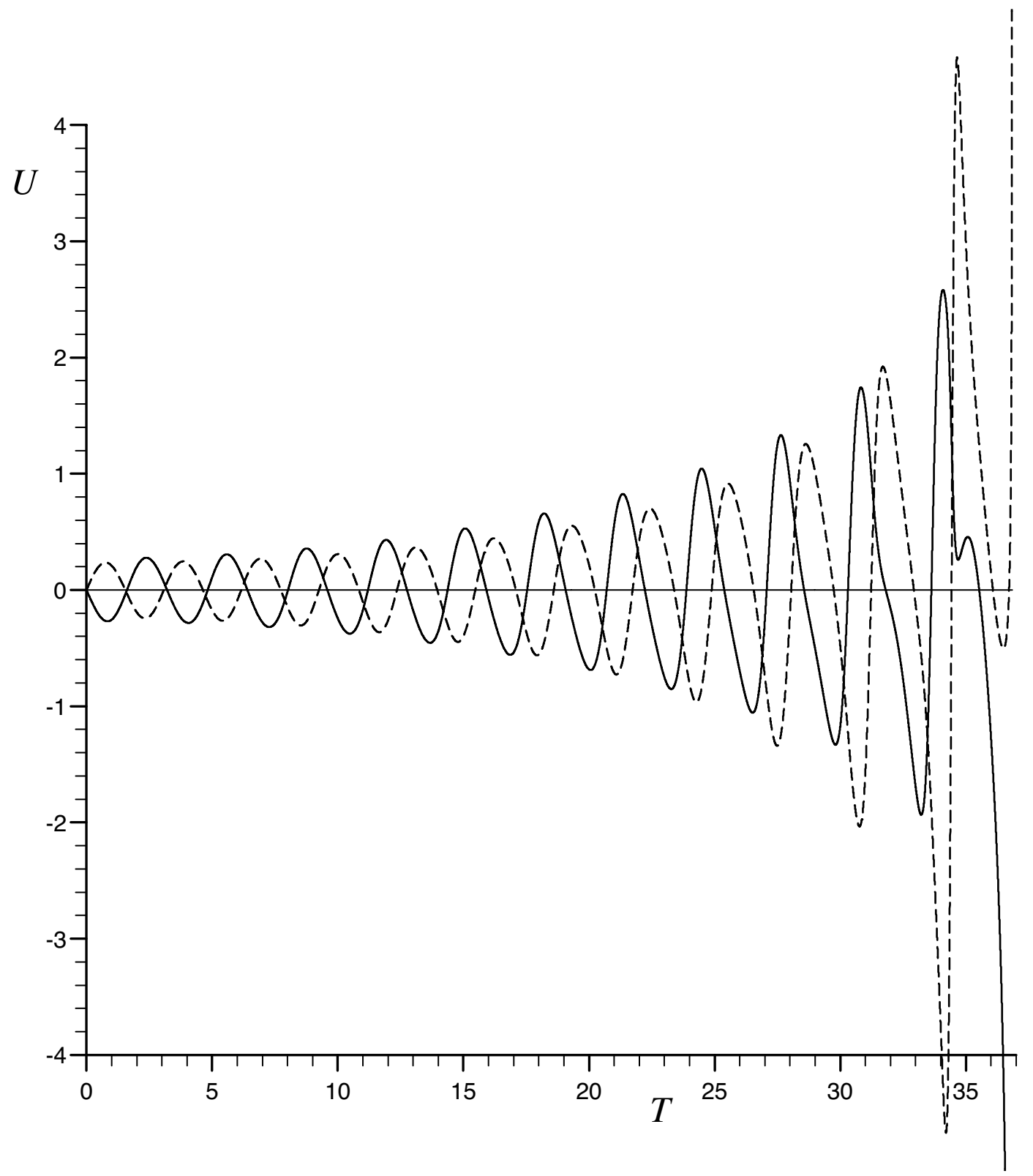

Figure 4: 
A.Kay: Figure 5

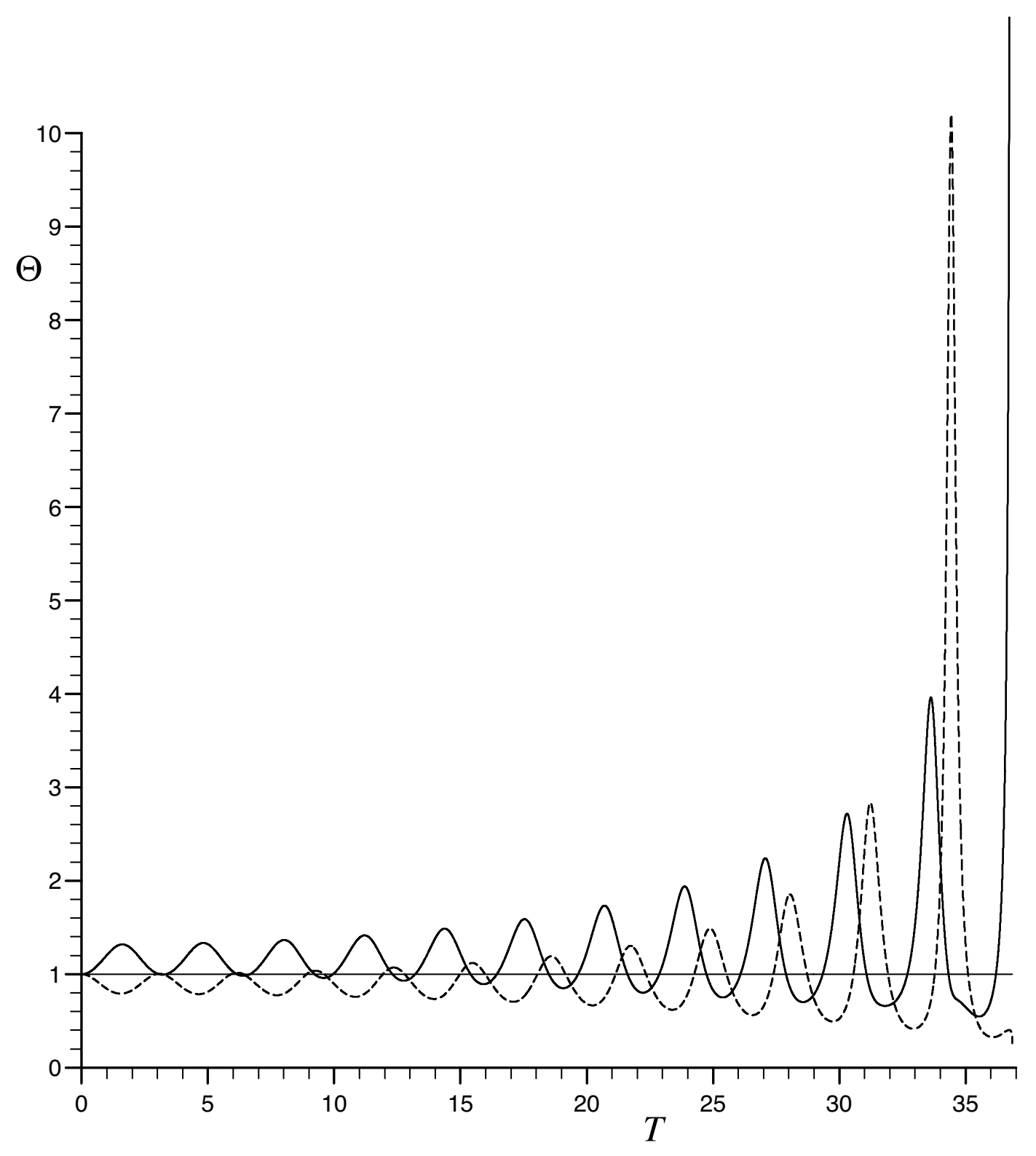

Figure 5: 